\title{
From Opportunistic Networks to 3GPP Network-Independent Device-to-Device Communication
}

\author{
Sylvia T. Kouyoumdjieva and Gunnar Karlsson \\ School of Electrical Engineering and ACCESS Linnaeus Center \\ KTH Royal Institute of Technology, Stockholm, Sweden \\ Email: \{stkou,gk\}@ee.kth.se
}

\begin{abstract}
Device-to-device (D2D) communication is being proposed as a complement to traditional communication via cellular networks. D2D establishes a direct communication link between mobile devices instead of relaying traffic through the cellular infrastructure. While D2D is currently one of the popular research topics in telecommunications, the concept of allowing devices in proximity to communicate directly with one another has been extensively studied for more than a decade under the name opportunistic communication. We here compare 3GPP's network-independent D2D communication to opportunistic communication and demonstrate that they share a number of common characteristics. We discuss which best practices from the opportunistic networking domain may be applied to networkindependent D2D, and outline open questions in the D2D design that have not been answered by previous research efforts.
\end{abstract}

\section{INTRODUCTION}

In cellular networks data traffic traverses the network infrastructure even when the communicating mobile devices are co-located and an alternative direct communication path could exist between them. Although cellular communication has apparent advantages for mobile operators such as explicit billing policies and full control over all communication in the network, it also has limitations. Firstly, communication via the cellular infrastructure may not always be the most efficient in terms of communication delay, power consumption and interference control. Furthermore, with the increase in mobile data traffic, and predictions for more than 24 exabytes $^{1}$ in monthly volumes by 2019 , operators are in search of auxiliary communication modes to support the traffic demands while decreasing the load on the infrastructure. Lastly, dependence on the cellular network implies that mobile communication is impossible when network coverage is unavailable due to expensive deployments or natural or man-made disasters.

To alleviate these limitations, device-to-device (D2D) communication has been proposed by the Third Generation Partnership Project (3GPP) as an alternative communication mode [1] to complement traditional communication over the cellular network. Essentially, device-to-device communication allows mobile devices to exchange data with one another over a direct communication link when in proximity instead of via the cellular network infrastructure. This communication mode

\footnotetext{
${ }^{1}$ An exabyte is a unit of information equal to one quintillion $\left(10^{18}\right)$ or, strictly, $2^{60}$ bytes.
}

is foreseen to improve spectrum reuse and decrease traffic volumes for the mobile operator, while at the same time increasing data rates, reducing end-to-end delays and decreasing energy consumption at the device end. Furthermore, deviceto-device communication is expected to be beneficial economically by providing new business opportunities in the area of proximity marketing and e-commerce, social networking, and entertainment.

D2D is not a new concept, though. The idea of establishing direct communication links between devices in proximity dates back to the introduction of mobile ad hoc networks (MANETs) in the 1990s, and has since then developed further in the areas of delay-tolerant networks (DTN), and most recently opportunistic networks. Due to their infrastructureless design, these networks have always been independent in operation of cellular networks. Only recently did researchers suggest that opportunistic networks can be used as a complement to cellular infrastructure in the context of mobile data offloading [2].

This article first provides an overview of modern deviceto-device communication as defined by 3GPP Release 12, and juxtaposes it to the well-studied field of opportunistic networks. It further outlines the findings in the area of opportunistic networks that can be used as bases for future D2D research, and also points out open questions that need further investigation.

\section{OpPortunistic Networks va 3GPP ProSe}

This section presents an overview of the fundamentals of opportunistic communication and device-to-device communication as defined by 3GPP Proximity Services (ProSe), and argues that opportunistic networks should be viewed as the predecessor of modern network-independent D2D.

\section{A. Opportunistic Networks}

For a decade academic research has explored a special class of mobile ad-hoc networks called opportunistic networks. The term opportunistic implies that there is not any knowledge of the network topology and connectivity graphs. Instead, communication opportunities arise as mobile nodes roam around in an area (according to some, often random, mobility pattern) and occasionally enter in direct communication range with one another. A node is considered to be a human or a 


\section{Communication via a traditional cellular network
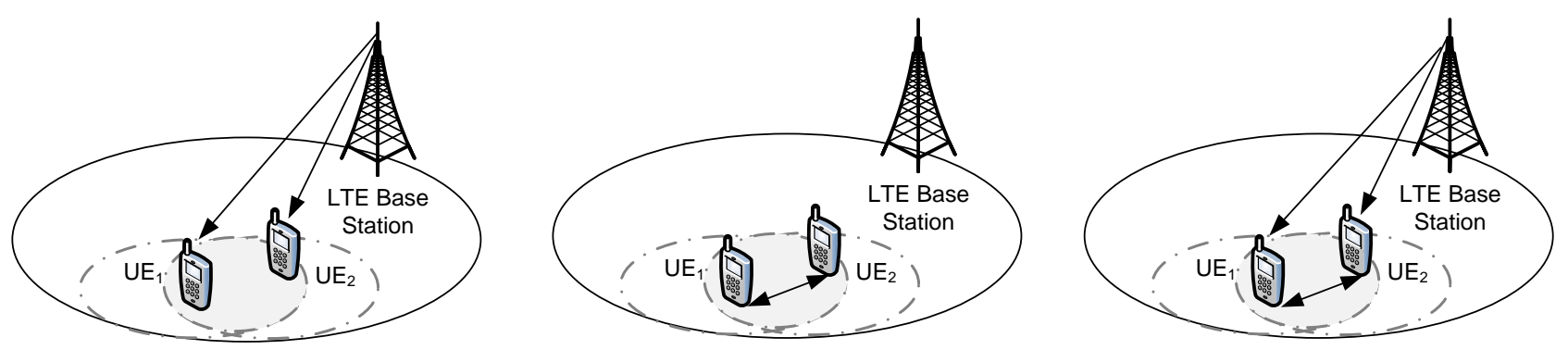

Fig. 1. In the presence of cellular infrastructure 3GPP's device-to-device communication combines the merits of traditional communication over the cellular network and infrastructureless opportunistic communication. When coverage is partial or absent, 3GPP's D2D resembles entirely opportunistic networking; however applications supported under partial coverage or no coverage at all only pertain to the public safety domain.

vehicle equipped with a device featuring at least one shortrange radio interface (i.e. a smartphone). The main motivation for such networks is content dissemination among nodes, with applications in the domain of mobile data offloading, public and road safety, and entertainment. Content creation can either be initiated by participants in the opportunistic network, or by authorized content producers on the Internet. Content dissemination thus depends on the popularity of contents, the node density and node mobility.

Opportunistic networks operate in the unlicensed spectrum utilizing either the $\mathrm{Wi}-\mathrm{Fi}$ or the Bluetooth radio interface currently available in modern devices. The cellular infrastructure is neither involved in conducting proximity estimations between device pairs nor in controlling connection parameters; thus opportunistic networks are truly network-independent by design. Instead, nodes periodically broadcast short beacon messages to announce their presence in the network. Association with suitable nodes in proximity is performed via a two-stage discovery process: a node discovery (identifying potential nodes in the vicinity) followed by a service discovery (identifying available applications on these nodes). A sample protocol for data dissemination in opportunistic networks is given in [3].

Due to the underlying mobility, opportunistic networks are characterized by short contact durations and high levels of churn. End-to-end connectivity among node pairs is often not possible to maintain. Most opportunistic networks are therefore considered to be content-centric instead of usercentric, shifting the focus from connecting a particular node pair to obtaining a particular content item (regardless of which node in the network is able to provide it).

\section{B. 3GPP's View on Device-to-Device Communication}

In 2013 device-to-device communication was added to Long Term Evolution (LTE) in 3GPP Release 12 within several work items related to $\mathrm{ProSe}^{2}$. The two main components in 3GPP ProSe are proximate discovery and communication.

Similar to opportunistic networks, ProSe discovery refers to the process of identifying whether two ProSe-enabled devices (called user equipments, UEs, in 3GPP language) are in proximity of one another; as opposed to opportunistic networks though, the discovery is suggested to happen in the licensed spectrum via the Evolved Universal Terrestrial Radio Access (E-UTRA) air interface [1]. Furthermore, proximity should be understood in a broader sense than simply physical distance. It may be determined based on channel conditions, signal-to-interference-and-noise ratio (SINR), throughput, and delay. ProSe communication refers to the actual data exchange between two ProSe-enabled UEs in proximity via a direct path over the E-UTRA or, in certain cases, a WLAN interface ${ }^{3}$; ProSe communication is not possible if ProSe discovery was unsuccessful.

From a UE perspective, discovery may be open or restricted, depending on whether a device can be discovered by a random neighboring node or not. From the viewpoint of the cellular network, discovery may be classified as network-assisted and network-independent. In network-assisted discovery, the responsibility of determining proximity between UEs belongs to the network; connection setup parameters, resource allocation and authentication are all issued by Evolved Packet Core (EPC) and communicated via the Evolved Node B (eNB) when nodes are in network coverage. UEs are thus expected to continuously report their locations to facilitate the proximity estimations at the network end. Regular location reporting may come at a high energy cost at the UE. Network-independent discovery assumes that UEs are capable of discovering nodes in proximity without communicating with the infrastructure

\footnotetext{
${ }^{2}$ Although the terms ProSe and D2D are often used interchangeably, ProSe is a general name used for describing the entire standardization package, while D2D defines the communication mode used for achieving proximity-based services.

${ }^{3}$ In [1] ProSe communication may also refer to the communication of two ProSe-enabled UEs whose data is locally routed via an eNB. In this article we only discuss ProSe communication in direct mode.
} 


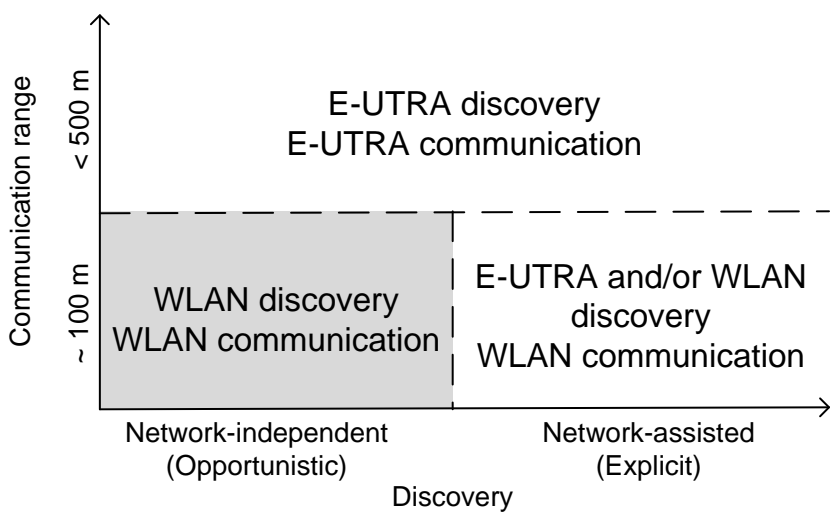

Fig. 2. Classification of 3GPP ProSe device-to-device communication with respect to the discovery process and the maximum communication range It can be seen that although opportunistic networks are only a subset of the current D2D proposal (lower left quadrant), the concept of opportunistic network-independent discovery propagates across communication ranges.

during the discovery process. However, the network is not entirely oblivious of the intention of UEs to engage in direct communication. Instead, available resources as well as authentication may be provided by the network.

Potential ProSe applications resemble applications developed for opportunistic networks, but categorized with respect to available network coverage. Most commercial applications, i.e., proximal e-marketing, social networking, and entertainment applications are envisioned to operate in network coverage. In this way, intermittent connectivity among mobile devices in the D2D domain may be compensated by continuous connectivity in the cellular domain allowing applications to also support real-time contents, for instance multiplayer gaming or video-on-demand. Pure network-independent operation is currently considered only for applications related to public safety [1].

\section{The Role of Opportunistic Networks}

Opportunistic networks and 3GPP's device-to-device communication paradigm have much in common (Table I). In fact, D2D represents a hybrid between traditional cellular networks and pure network-independent opportunistic networks, Fig. 1. A closer examination however reveals that opportunistic networking encompasses a subset of the use-cases instrumented in 3GPP ProSe. Fig. 2 illustrates the positioning of opportunistic networks in the ProSe domain. Note that the change in communication range only reflects the wireless technology used for discovery and communication. Although current applications based on the opportunistic networking paradigm operate in unlicensed spectrum, the underlying principles of opportunistic networking are independent of the wireless technology used. Thus, opportunistic networks should be considered as the origin of modern network-independent device-to-device communication.

\section{Design Aspects: Lessons LeARned AND OpEn QUESTIONS}

In this section we examine in more detail the main design aspects of network-independent D2D communication as
TABLE I

COMPARISON OF FUNDAMENTAL DESIGN FEATURES FOR 3GPP PROXIMITY-BASED SERVICES AND OPPORTUNISTIC NETWORKS.

\begin{tabular}{|c|c|c|c|}
\hline \multicolumn{2}{|c|}{ Design Aspects } & 3GPP ProSe & Opportunistic \\
\hline Discovery & $\begin{array}{l}\text { Direct } \\
\text { Network-assisted }\end{array}$ & $\begin{array}{l}\sqrt{ } \\
\sqrt{ }\end{array}$ & $\sqrt{ }$ \\
\hline Discovery & $\begin{array}{l}\text { Synchronous } \\
\text { Asynchronous }\end{array}$ & $\begin{array}{l}\sqrt{ } \\
\sqrt{ }\end{array}$ & $\begin{array}{l}\sqrt{ } \\
\sqrt{ }\end{array}$ \\
\hline Spectrum & $\begin{array}{l}\text { Licensed band } \\
\text { Unlicensed band }\end{array}$ & $\begin{array}{l}\sqrt{ } \\
\sqrt{ }\end{array}$ & $\sqrt{ }$ \\
\hline Mobility & UE-UE link & $\sqrt{ }$ & $\sqrt{ }$ \\
\hline Application & $\begin{array}{l}\text { Delay-tolerant } \\
\text { Real-time }\end{array}$ & $\sqrt{ }$ & $\sqrt{ }$ \\
\hline Paradigm & $\begin{array}{l}\text { User-centric } \\
\text { Content-centric }\end{array}$ & $\begin{array}{l}\sqrt{ } \\
\sqrt{ }\end{array}$ & $\sqrt{ }$ \\
\hline Operation & $\begin{array}{l}\text { In coverage } \\
\text { Out of coverage }\end{array}$ & $\begin{array}{l}\sqrt{ } \\
\sqrt{ }\end{array}$ & $\begin{array}{l}\sqrt{ } \\
\sqrt{ }\end{array}$ \\
\hline Outreach & $\begin{array}{l}\text { Single hop } \\
\text { Relay }\end{array}$ & $\begin{array}{l}\sqrt{ } \\
\sqrt{ }\end{array}$ & $\begin{array}{l}\sqrt{ } \\
\sqrt{ }\end{array}$ \\
\hline
\end{tabular}

defined by 3GPP ProSe, and we discuss how these aspects have been addressed in the field of opportunistic networking. We evaluate which of the available solutions can be adopted directly in the ProSe design, and which areas are still open for further investigation. We approach the discussion from a system viewpoint. A radio access perspective can be found in [4]; security and privacy aspects are explored in [5], [6].

\section{A. Continuous vs Energy-efficient Discovery}

Due to the sporadic nature of contacts in opportunistic networks, continuous discovery is often considered: nodes should have their radio interfaces constantly turned on in order to be able to discover neighboring devices in a quick and reliable manner. Since nodes are battery-powered, the energy consumption during discovery should be minimal. The energy consumed by a radio interface can be divided into two main components: energy spent in idle state (when a node is listening for incoming signals from devices in proximity), and energy spent in busy state (when a node is sending or receiving data on the radio interface). With most current technologies operating in the unlicensed spectrum the energy spent in both states is of the same order of magnitude making discovery as costly as actual data communication.

To achieve energy-efficient discovery, the radio interface often needs to be duty cycled, alternating between periods when it is turned on and periods when it is turned off. Depending on the distribution of "on" and "off" durations as well as the network dynamics, turning the radio interface off may come at a cost of lost contact opportunities.

1) Synchronous vs Asynchronous Schemes: Synchronous discovery schemes assume that all nodes in the network turn on their radio interface simultaneously. Theoretically this often makes these schemes more appealing than asynchronous ones since they offer faster and more reliable discovery at a potentially lower energy cost. In reality however the energy consumption is not bound to the type of discovery scheme but to the time during which the radio interface is turned on. In fact, a duty-cycled asynchronous scheme would consume exactly the same amount of energy as a synchronous one as long as they are parameterized with the same distribution of listening durations and operate with the same underlying 
wireless technology. However, the time it would take for a node to discover its neighbors in an asynchronous manner would be generally longer than if a synchronous scheme is adopted.

An open question is how fast discovery should be performed. For static and semi-static environments, speed of discovery may not be essential while for dynamically changing environments, fast discovery may be critical for good performance. Ultimately, the discovery scheme may depend on the class of applications that it is serving.

2) Licensed vs Unlicensed Spectrum Operation: Opportunistic networks have been suggested to work in the unlicensed spectrum, partially due to the fact that researchers only had access to radio technologies operating in the industrial, scientific and medical (ISM) radio band such as Wi-Fi and Bluetooth. Operating in unlicensed spectrum comes at a cost of uncontrolled interference and degraded performance in densely populated areas. However it has one major advantage: all devices can participate in the opportunistic network and freely exchange data with one another. 3GPP suggests that D2D discovery and communication should instead be done primarily in the licensed spectrum in order to avoid interference issues. However, operation in licensed spectrum is not trivial for devices subscribed to different mobile operators. Whether a direct D2D discovery and communication would be possible across operators depends strongly on the agreements between operators. If the agreements are not in place, D2D may become only prerogative to clients of a particular mobile operator, thus creating strong segmentation in the available D2D services across operators.

3) The Battle of the Directs: With the introduction of LTEDirect [7] in Release 12, it was quickly proclaimed to be the preferred candidate for direct device-to-device discovery and communication due to its longer communication range, low energy consumption and in-band operation. A recent study shows that LTE-Direct is better suited for opportunistic discovery than any of the available technologies in the unlicensed spectrum such as Wi-Fi Direct [8] or Bluetooth Low Energy [9]. However, an actual comparison between these technologies is hard to justify. On the one hand, WiFi Direct was historically developed as an alternative to Bluetooth, allowing for longer communication ranges and higher data rates between device pairs. The standard was never intended for opportunistic discovery of random contacts in urban areas. This can be inferred from the required manual pairing before connection establishment as well as the slow two-step discovery process [10]. On the other hand, LTEDirect is developed solely for the purpose of direct discovery of sporadic contacts opportunities in urban environments. It features a duty-cycling scheme in which the radio interface is only turned on for a small interval of time in the beginning of each cycle (Fig. 3) while the long communication range (up to $500 \mathrm{~m}$ ) augments the micro-level mobility and assures that any two UEs in proximity will be able to discover each other within a single cycle.

Due to the fundamental differences of Wi-Fi Direct and LTE-Direct, Release 12 explicitly differentiates between their roles for future device-to-device communication. The main use

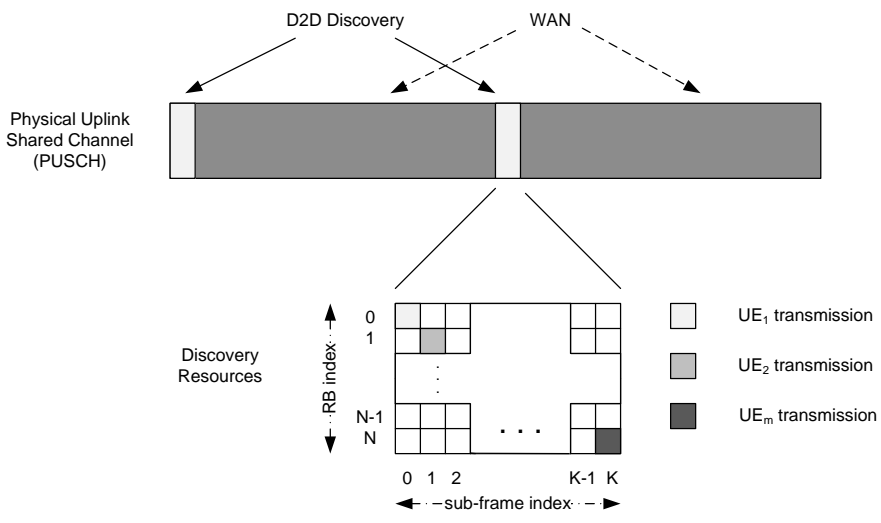

Fig. 3. Allocation of discovery resources in LTE Direct.

case for Wi-Fi Direct (as well as other future WLAN technologies) is as a supporting technology for ProSe communication between device pairs in close proximity while LTE Direct finds application in both network-independent and network-assisted D2D discovery and communication.

We note that Wi-Fi Aware [11] promises to enable real proximity-based services in the unlicensed spectrum. Since the standard is relatively new, it is yet not defined how it will fit in the D2D landscape of radio technologies. Thus, it is still an open question which is the most appropriate technology for performing opportunistic discovery and communication, and whether there will be a plethora of technologies to cater to the needs of D2D.

\section{B. Push vs Pull-Based Discovery Mechanisms}

Since opportunistic networks assume no previous knowledge of the environment, discovery is suggested to be pushbased. In push-based discovery, nodes periodically broadcast their presence in the network ("I am here" type of message). Based on application-specific criteria, nodes that receive this broadcast message can then decide whether to initiate communication with the announcing node. Push-based mechanisms are appropriate for content-centric networks in which the emphasis is on obtaining particular data regardless of the source that provides it.

3GPP ProSe defines two possible discovery mechanisms, or models: a push-based model, similar to the one used in opportunistic networks, and a pull-based model, where an UE requests information about discoverable devices ("Are you there?" or "Who is there?" type of message) [5]. Both mechanisms can be used in network-independent discovery, however they serve different purposes. As with opportunistic networks, push-based discovery is intended for usage in content-centric environment. Pull-based models, on the other hand, serve two different purposes depending on the question they address in the discovery message: In the first case, the discovering node is expected to know explicitly whom it is trying to reach, and thus it is better suited for user-centric networks, i.e. for network-assisted discovery when the network initiates a D2D connection among a particular pair of UEs. However, pull-based models can also be adopted in networkindependent discovery when addressing the question "Who is 
there?". In the context of opportunistic discovery for D2D communication, the main difference between a push- and pullbased discovery mechanism is in the commercial use cases that they target: push-based discovery mechanisms are appropriate for e-commerce applications (i.e. a coffee shop announcing its presence in the network), while pull-based discovery models can be used by UEs actively searching for information in their vicinity (i.e. a user who is interested in locating all coffee shops in her vicinity).

\section{Effect of Mobility}

Potential device-to-device communication opportunities occur when two or more UEs come in range for direct communication. The physical duration of a contact depends on a number of factors, among which are the communication range and the mobility. 3GPP suggests that at least three communication range classes should be defined: short, medium and maximum, however their exact values are not specified yet. In each of these classes the effect of mobility would be different.

With the out-of-band radio technologies currently available in smart mobile devices, opportunistic networks are bound to operate within a short communication range (in the order of tens of meters). This has lead to exhaustive research efforts in the area of micro-level mobility modeling in order to capture precisely even the shortest contact opportunities with duration of only few seconds among proximate devices [12], [13]. With the introduction of longer communication ranges in $3 \mathrm{GPP}$ ProSe (up to $500 \mathrm{~m}$ ) resulting in longer potential contact durations, examining micro-level mobility may not be of primary concern for D2D. However, most mobility models can easily be adapted to the context of 3GPP ProSe.

Mobility and Relaying: One of the suggested applications for D2D is in scenarios with partial coverage where ProSeenabled UEs act as relays connecting out-of-coverage nodes with infrastructure or establishing a link between two out-ofcoverage UEs which are not in direct communication range with one another (Fig. 4). Relaying is a natural extension of D2D communication when nodes are static. In the presence of mobility however relaying is not trivial. Links may break and new communication paths may be established with time; nodes may enter in and out of coverage of infrastructure or in communication range with a more appropriate relay candidate (i.e. based on delay or SINR values). It has been previously shown that in the presence of mobility relaying may not be suitable due to the high dynamics in the network topology [13]. Thus, defining algorithms and protocols for performing relaying in $\mathrm{D} 2 \mathrm{D}$, especially when nodes are outof-coverage, is still an open issue.

\section{User and Application Identities}

Regardless of the discovery mechanism (push-based or pull-based) in opportunistic networks the discovery process usually consists of two stages: a node discovery (identifying potential nodes in the vicinity) followed by a service discovery (identifying available applications on these nodes). Thus, two separate identities are required for discovering nodes and services. A node identity uniquely identifies a single device in

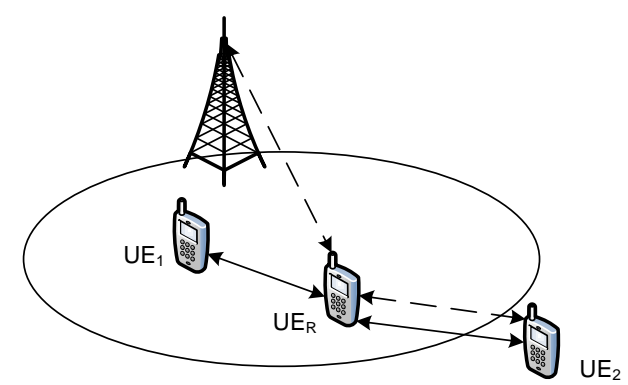

Fig. 4. Establishing a communication link between two UEs via a relay node (UE-to-UE relay) and between the network and an out-of-coverage UE via a relay node (UE-to-Network relay) under partial network coverage. A UE-to-UE relay is also possible when all ProSe-enabled devices engaged in communication are out-of-coverage.

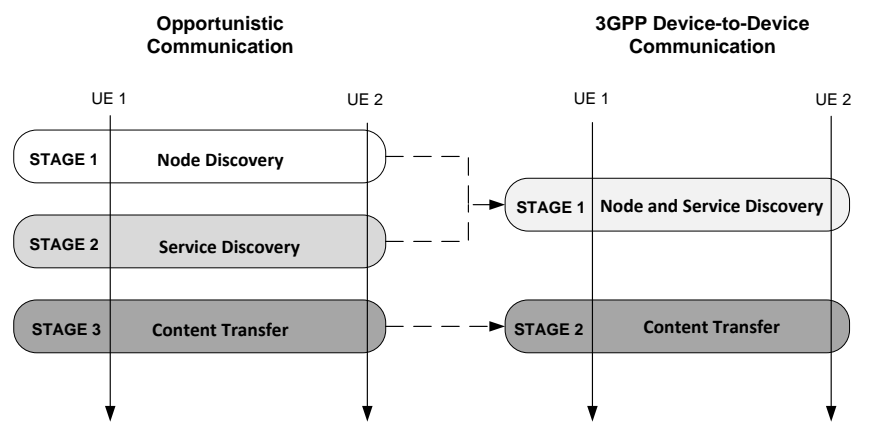

Fig. 5. In 3GPP ProSe the device and application discovery are consolidated into a single discovery message. The actual data communication protocol however is still undefined. Communication protocols designed for opportunistic networks may also find application in D2D.

an opportunistic network. Often a node identity is considered to be simply the Layer 2 address of the device. A service identity describes a particular class of information, for example news or traffic updates. A service identity is not bound to a particular application.

3GPP extends the node and service identifiers, introducing a total of four identities to facilitate node and service discovery. (1) A ProSe application identity is used to uniquely identify a single application across the Public Land Mobile Network (PLMN). (2) An application user identity extends the application identity and binds it to a specific device; the network can then have a direct mapping between applications and devices on which they are available. From a device perspective, (3) a ProSe UE identity uniquely identifies a single device. Finally, (4) the ProSe discovery code, sometimes referred to as an expression, is used by each UE for announcing its identity (and corresponding applications) to devices in proximity; the ProSe discovery code can be dynamically updated by the network. The expression may be private (only accessible by a group of subscribers), or public. Similar to the service identity in opportunistic networks the expression is also used to decouple data from its corresponding application. As a typical example, if a user is interested in photography, she can find a new exhibit opening (broadcast via Yelp), an local photography business announcement (broadcast via LinkedIn), or another photography expert (broadcast via Twitter). 
Fig. 5 illustrates the discovery and communication process in opportunistic and 3GPP D2D networks. At first it may seem that the introduction of expressions in 3GPP D2D makes discovery faster and more efficient since it allows simultaneous discovery of devices and the corresponding services that run on them. However, consolidating node and service discovery information into a single frame is a design choice, and as such can be applied to opportunistic and 3GPP D2D networks alike. For instance, Bloom filters have been suggested in opportunistic networks for accommodating more information into a single frame. In fact, the new four-tier identity system of 3GPP's ProSe raises many practical questions. The introduction of ProSe application identities suggests that operators aim at gaining control over the management of proximity-based applications. However, since ProSe application identities are defined in the scope of an operator's network, this may lead to fragmentation of services across operators. Furthermore, it is unclear whether it is feasible (or even needed) to support unique ProSe application identities for all available applications. Finally, mapping application to device identities may be considered as a privacy intrusion.

\section{E. Data Communication}

In opportunistic networks communication among devices in proximity is an extension of the discovery process. If both device and service discovery are successful, devices simply establish a unicast communication to discover and potentially download contents of interest (Fig. 5). Since communication and discovery utilize the same radio resources, data rates over direct links between devices depend on environmental conditions such as node density, interference as well as mobility.

Direct data communication in D2D can be either networkindependent or network-authorized, however networkindependent D2D communication is currently only applicable to authorized ProSe-enabled UEs for public safety. Thus, for commercial applications, even though discovery can be purely opportunistic, communication between proximate devices is not possible without network control. Instead, once devices in proximity have discovered one another, a network authorization procedure is required. The network authorization procedure determines whether to provide authorization keys to the UEs and to establish EPS bearers. De facto, even if D2D discovery is successful, there is no guarantee that the UEs will actually communicate over a direct link. Whether communication would then be transferred back over the cellular infrastructure, or it would be postponed or denied is not yet defined.

Data structures: Motivated by peer-to-peer networks, a common practice in opportunistic networks is to divide large content items into smaller pieces, called chunks, to allow for utilization of short contact opportunities between mobile devices, as well as downloading contents from multiple sources (simultaneously or successively). In-band D2D communication in $3 \mathrm{GPP}$ provides extended communication range (thus longer contact durations under the same underlying mobility) as well as higher data rates; however the concept of chunks may still be applicable if contents can be downloaded from multiple sources or at multiple stages.

\section{F. Application Domain}

Due to the infrastructureless design of opportunistic networks, applications are predominantly dealing with delayinsensitive data in the context of information dissemination. In general, applications can be divided into two main categories with respect to the context in which they are used: urban area applications and challenged area applications. Urban area applications assume that opportunistic communication occurs among nodes in network coverage and is used as a complement or alternative to traditional communication via the cellular network. Typical examples of such applications are proximal social networking [14], proximal entertainment [15], [16] and mobile data offloading [2]. These applications require a critical mass of participating devices, and are often motivated by providing enhanced add-on services on top of traditional cellular infrastructure. Challenged area applications, on the other hand, are usually suggested as an alternative communication mode in disaster scenarios, in developing regions lacking a cellular infrastructure or in the presence of governmental oppression.

Due to its longer communication ranges, D2D adds traditionally centralized applications such as location-based marketing and e-commerce to the range of potential proximal services. Furthermore, applications are now not only limited to exchanging delay-tolerant data but can instead support realtime contents for entertainment applications such as multiplayer gaming. The two main obstacles preventing realtime communication in opportunistic networks, short contact durations and high churn levels (due to mobility), are easier to overcome in 3GPP: if a direct link breaks, an alternative path can seamlessly be established over the network infrastructure.

\section{G. Privacy}

Opportunistic networks are designed to preserve node privacy since the network has no control over the established communication paths or the data that is transferred over these paths. Furthermore, as discovery is network-independent, node location is also private. In 3GPP, even when D2D discovery is network-independent, D2D communication is expected to be network-authorized. This reveals the communicating pairs as well as their locations and (possibly even) intended contents to share. Undoubtedly this should raise concerns among users who are not willing to share their location and communication patterns with the operator at all times.

\section{Discussion AND SUMmary}

In the early 2000s researchers proposed opportunistic communication utilizing direct links between devices, co-located in space and time, as an alternative to traditional cellular communication. The lack of appropriate radio technology, the unsuitable branding with respect to potential applications (i.e., sharing copyrighted contents) as well as the absence of clear business use-cases made the concept unattractive to mobile operators. Today, 3GPP has successfully identified these shortcomings, designing network-independent D2D as an operator-friendly version of opportunistic networking. Device-to-device communication was introduced in Release 12 of 3GPP's specification for Proximity-based Services with 
the promise to improve spectrum utilization, throughput and energy consumption while at the same time relieving the traffic volumes on the operator side. Although the main focus currently is the applicability of D2D to the public safety domain, commercial usage is envisioned in forms of proximate marketing and e-commerce.

In this paper we reasoned why opportunistic networks should be considered as a predecessor of network-independent D2D communication by extensive comparison of design features of the two solutions. We further discussed some of the newly-raised challenges for $\mathrm{D} 2 \mathrm{D}$, namely issues related to technologies, scalability and privacy. Given the current state of the specification it is still unclear whether 3GPP's D2D would become a universal solution or if infrastructureless opportunistic networks will be able to co-exist with it in the future.

\section{REFERENCES}

[1] 3rd Generation Partnership Project: Technical Specification Group Services and System Aspects, "Feasibility study for Proximity Services (ProSe), Release 12, TR 22.803 ver. 12.2.0," June 2013.

[2] B. Han, P. Hui, V. Kumar, M. Marathe, J. Shao, and A. Srinivasan, "Mobile data offloading through opportunistic communications and social participation," Mobile Computing, IEEE Transactions on, vol. 11, no. 5, pp. 821-834, May 2012.

[3] Ó. Helgason, E. A. Yavuz, S. Kouyoumdjieva, L. Pajevic, and G. Karlsson, "A mobile peer-to-peer system for opportunistic content-centric networking," in Proc. ACM SIGCOMM MobiHeld workshop, 2010.
[4] X. Lin, J. Andrews, A. Ghosh, and R. Ratasuk, "An overview of 3gpp device-to-device proximity services," Communications Magazine, IEEE, vol. 52, no. 4, pp. 40-48, April 2014.

[5] 3rd Generation Partnership Project: Technical Specification Group Services and System Aspects, "Study on architecture enhancements to support Proximity-based Services (ProSe), Release 12, TR 22.703 ver. 12.0.0," February 2014.

[6] 3rd Generation Partnership Project: Technical Specification Group Services and S. Aspects, "Study on Security issues to support Proximity Services (ProSe), Release 13, TR 33.833 ver. 1.3.0,” January 2015.

[7] Qualcomm Technologies Inc., "Lte direct: The case for device-to-device proximate discovery," February 2013.

[8] Wi-Fi Alliance, "Wi-fi peer-to-peer (p2p) technical specification v1.5."

[9] Qualcomm, "Lte-direct trial," Tech. Rep., February 2015.

[10] D. Camps-Mur, A. Garcia-Saavedra, and P. Serrano, "Device-to-device communications with wi-fi direct: overview and experimentation," Wireless Communications, IEEE, vol. 20, no. 3, pp. 96-104, June 2013.

[11] Wi-Fi Alliance, "Wi-fi aware: Better proximity technology for personalized experiences," July 2015.

[12] P. Pirozmand, G. Wu, B. Jedari, and F. Xia, "Human mobility in opportunistic networks: Characteristics, models and prediction methods," Journal of Network and Computer Applications, vol. 42, no. 0, pp. 45 $-58,2014$.

[13] Ó. Helgason, S. T. Kouyoumdjieva, and G. Karlsson, "Opportunistic communication and human mobility," Mobile Computing, IEEE Transactions on, vol. 13, no. 7, pp. 1597-1610, July 2014.

[14] S. Kouyoumdjieva, E. A. Yavuz, Ó. Helgason, L. Pajevic, and G. Karlsson, "Opportunistic content-centric networking: The conference case demo," demonstration at IEEE Infocom, 2011.

[15] Z. Chen, E. Yavuz, and G. Karlsson, "What a juke! a collaborative music sharing system," in Proc. IEEE WoWMoM, June 2012, pp. 1-6.

[16] I. Marfisi-Schottman, G. Karlsson, and J. C. Guss, "Demo: Opphos - a participative light and sound show using mobile phones in crowds," in Proc. ExtremeCom, August 2013. 